\title{
Outcome of carpal tunnel release surgery in patients with diabetes
}

\section{David M. Pagnanelli, M.D., and Steven J. Barrer, M.D.}

\section{Division of Neurosurgery, Abington Memorial Hospital, Abington, Pennsylvania}

The surgical outcomes in 149 patients with diabetes and carpal tunnel syndrome who underwent transverse carpal ligament release surgery are reported. Associated factors such as insulin dependence, length of time with diabetes, electrodiagnostic studies, severity of neurological deficit, presence of polyneuropathy, and cervical disc disease were considered. Analysis of preoperative and postoperative symptoms, clinical findings, diagnostic studies, and patient self-assessment indicated that the majority of patients with diabetes experienced a favorable surgical outcome, regardless of any associated factors. Eighty-four percent of the patients reported good to excellent postoperative results in their hands. Further analysis of the subpopulation of insulin-dependent diabetics showed that $81 \%$ experienced good to excellent postoperative results. These results compare favorably with those of the control group: 200 nondiabetic patients, of whom $90 \%$ rated their results from carpal tunnel release surgery as good to excellent. There were no major complications in any group.

Key Words * carpal tunnel syndrome * carpal tunnel release surgery * diabetes * polyneuropathy

Although patients with diabetes have increased susceptibility to developing compressive neuropathy,[5] the mechanism of the susceptibility remains controversial. Experimental studies evaluating alterations of fast axoplasmic flow and blood flow through small caliber vessels are inconclusive.[2,4,9,11,14,15,17] We report the results of our extensive experience in the operative treatment of the most common compressive neuropathy, carpal tunnel syndrome (CTS). Surgical outcome was evaluated by using specific factors, which included insulin and noninsulin dependence, length of time dependent on insulin, severity of preoperative neurological deficits, severity of preoperative electromyographic (EMG) changes, and the presence of associated factors, such as cervical disc disease, cervical myelopathy, cervical radiculopathy, diabetic peripheral neuropathy, multiple entrapment neuropathies, hypothyroidism, and arthritis.

\section{CLINICAL MATERIAL AND METHODS}

From 1982 to 1997 we performed approximately 3612 surgeries for carpal tunnel release (CTR). Clinical information about this patient population, collected in our computerized database, included the original history and physical examination, notes on subsequent patient visits, electrodiagnostic studies, and other pertinent tests. Additional data were collected from questionnaires (used 1 week, 1 month, 6 months, and 1 year postsurgery) that recorded patients' estimations of their preoperative pain, paresthesias, functional loss, and postoperative outcome. Only patients with completed files at 6 months and beyond were entered into this study. An age- and sex-matched cohort of patients with CTS was selected from the 
computer database to compare surgical outcomes of patients with and without diabetes.

Information on 200 consecutive age-matched nondiabetic patients was obtained from our database. The average age of this group was 57.7 years. The female/male ratio was 2.1:1, and the average duration of symptoms was 10 years. One hundred eighteen of these patients had symptoms and electrodiagnostic studies in both hands that were positive for CTS.

The results of surgery for patients with and without diabetes were determined from our clinical evaluation at follow-up visits and by patient input on questionnaires. The patients used a rating system of "excellent," "good," "fair," and "poor" to describe relief of preoperative symptoms, which included intermittent daily paresthesias, nocturnal paresthesias, pain, and improvement in hand function. Complete data collected 6 months postsurgery were available on 1599 patients, who had undergone 2445 CTR surgeries.

Two hundred thirty-five CTR surgeries were performed in the 149 patients with diabetes mellitus. Prior to surgery muscle atrophy was demonstrated in 63 hands (27\%), hand weakness in $122(52 \%)$, and nocturnal paresthesias in $195(83 \%)$.

In the 149 patients with diabetes, the female/male ratio was $2.4: 1$, the average age of the population was 59 years, and the average duration of symptoms was 5 years. The average length of time that the patient had been diabetic was 13.8 years. Forty-six of the 149 patients were dependent on insulin. In the insulin-dependent subgroup the average age was 55.7 years, the duration of symptoms was 5 years, and length of time dependent on insulin was 10 years.

Electrodiagnostic studies demonstrated criteria for bilateral CTS in 143 of 149 diabetic patients. Of these patients, 87 showed clinical symptoms bilaterally and underwent bilateral CTR surgery.

Electromyographic and nerve conduction studies revealed abnormal results in all of the patients except one who refused the studies.

Multiple nerve compression abnormalities were common in this population of diabetic patients. In addition to CTS, patients had the following associated problems: cervical radiculopathy; ulnar nerve compression at the elbow and bilaterally; and diabetic polyneuropathy, defined as axonal injury or denervation in multiple distal areas of peripheral nerves other than those sites associated with common compression syndromes.

The surgical technique we used[12] is a modification of the technique described originally by Paine.[13] A small transverse incision is made on the ulnar side of the distal wrist crease. The subcutaneous tissue is retracted and the transverse carpal ligament is exposed. The ligament is opened in the direction of its fibers and the underlying structures are identified. The retinaculatome is then introduced into the wound with the foot plate of the instrument beneath the carpal ligament. The elevated knife blade cuts the ligament when the retinaculatome is advanced into the palm in a slightly ulnar direction. It is then turned around and advanced up into the forearm to divide the ligament completely. A long thin retractor blade is introduced so that the cut edges of the entire ligament can be inspected under loupe magnification.

\section{RESULTS}

We obtained complete 6-month postoperative data on 213 hands in 149 diabetic patients undergoing surgery for CTR. Nocturnal paresthesias abated in 196 hands (92\%), complete resolution of preoperative numbness was attained in 132 hands (62\%), and improvement in hand function was confirmed in 188 
hands (88\%). Patients reported an excellent result in 112 hands (53\%), a good result in 67 hands (31\%), a fair result in 19 hands (9\%), and a poor result in 15 hands (7\%) (Fig. 1).

\section{Diabetics vs Nondiabetics}

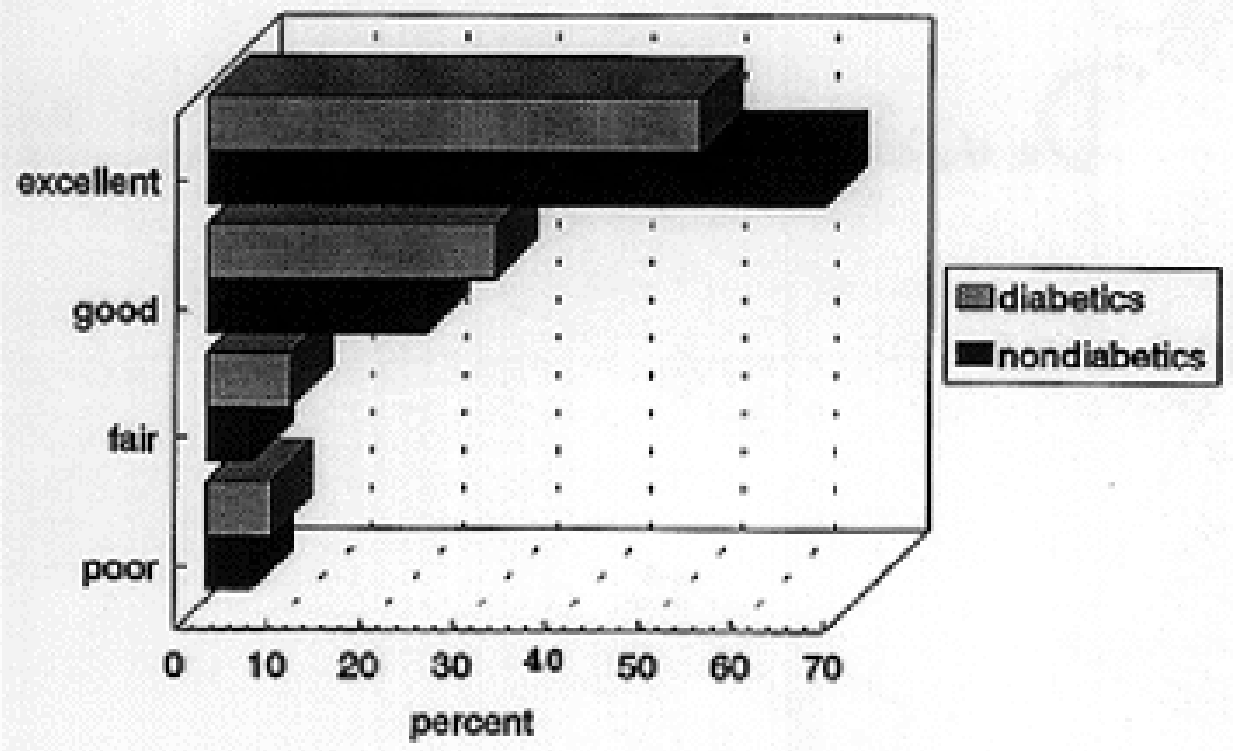

Fig. 1. Graph showing a comparison of patient self-assessed surgical outcome in diabetic and nondiabetic patients 6 months after CTR surgery.

Of the 19 hands with a fair result, 11 had complete relief of nocturnal paresthesias and improvement in strength and sensation. Five hands had superficial infections after surgery that were resolved by administration of antibiotic drugs. One patient complained of left-hand swelling, which was relieved by an injection of a steroid agent. Another patient had residual left-hand problems, primarily arthritic in origin, which showed improvement following the use of a wrist splint and ibuprofen. Two of these fair results were reported by a physician with juvenile-onset diabetes who had had the disease for 33 years. He suffered severe diffuse polyneuropathy and noted only minimal improvement in sensation.

Of the 15 hands with poor results, complete relief of nocturnal paresthesias was achieved in six. Of these patients with one or more hands having a poor result, one also had an underlying cervical myelopathy and another, an insulin-dependent diabetic for 17 years, refused preoperative electrodiagnostic studies and was not compliant with blood glucose monitoring. Patients reported that hand numbness was not as severe, despite underlying arthritic changes, in the remaining nine hands with poor results.

As is common in patient surveys, the patient's expectations may not be the same as those of the surgeon. A realistic postoperative outcome in many of our patients may simply be relief of intermittent or nocturnal paresthesias and not resolution of their preoperative neurological deficit. Nine of the hands rated fair and five rated poor had been the subject of mitigating preoperative circumstances. In spite of this, 14 patients whose hands were rated in the fair and poor groups were relieved of their nocturnal and intermittent paresthesia and claimed improvement in their preoperative numbness.

Of the 69 hands from 46 insulin-dependent patients, the outcome was rated excellent in 34 (49\%), 22 $(32 \%)$ hands were rated as good, seven $(10 \%)$ were rated as fair, and six $(9 \%)$ were rated as poor. In 
total, $56(81 \%)$ of 69 hands were rated as being a satisfactory surgical result (Fig. 2).

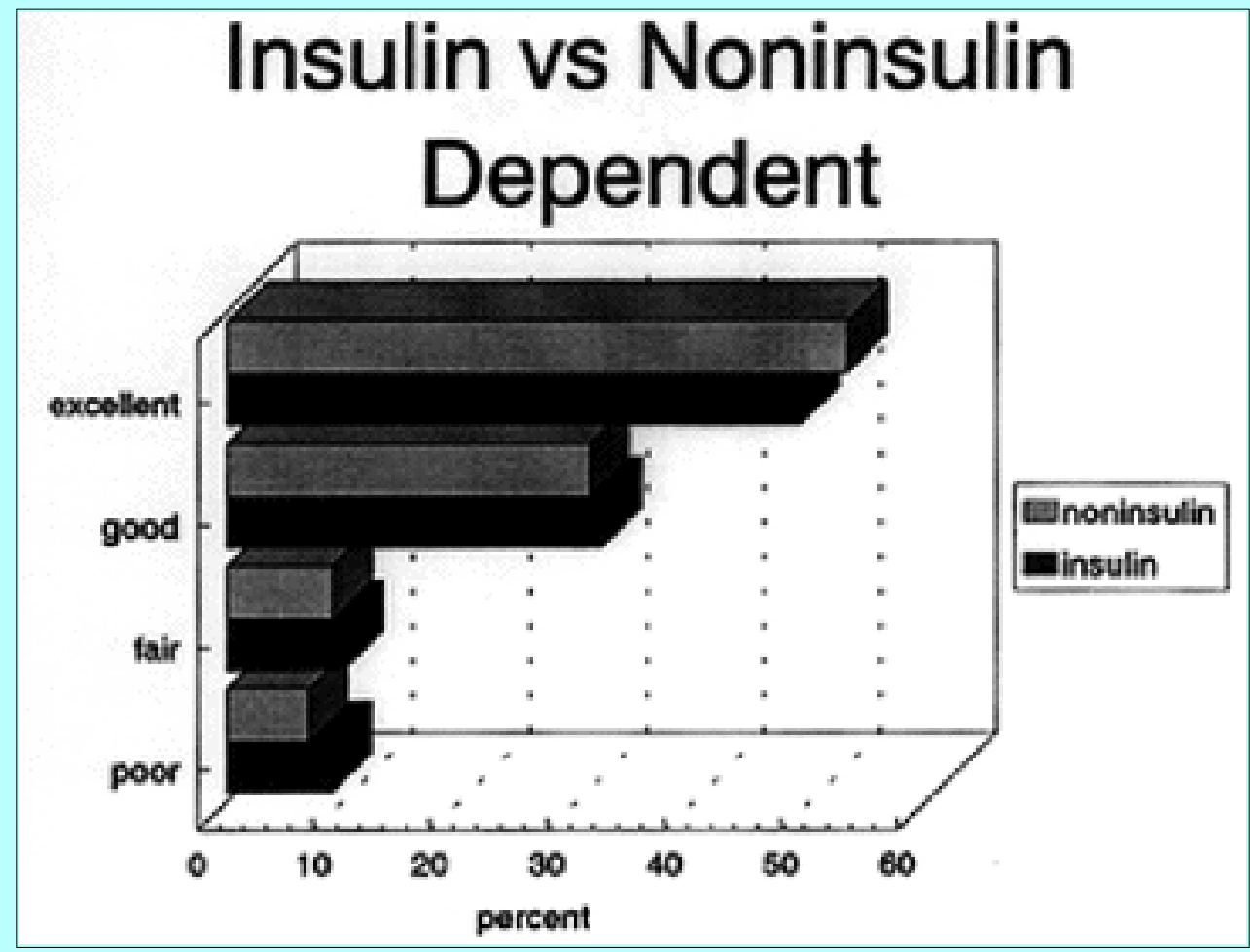

Fig. 2. Graph showing a comparison of patient self-assessed surgical outcome in insulin-dependent and noninsulin-dependent diabetic patients 6 months after CTR surgery.

Three hands in which there were superficial wound infections were treated by warm soaking; the patients received oral antibiotic medications and rated the outcome of their surgeries as good on the questionnaire. No injuries to the median nerve or any of its branches were noted.

The results in the 200 age-matched patients without diabetes (the control group) were very similar to the results we reported on for 577 patients in 1991.[12] Of the 118 patients who displayed symptoms and were found to have positive electrodiagnostic studies in both hands, 105 underwent bilateral CTR surgery during a single session in the operating room. Carpal tunnel release surgery was performed in 305 hands in this group.

Associated systemic disorders noted in the control group included arthritis, hypothyroidism, cervical spondylosis, cervical spinal cord tumor, myasthenia gravis, polyarteritis, and amyloidosis.

During the initial visit the examining physician found that 56 hands $(18 \%)$ in patients without diabetes had thenar muscle atrophy, 235 hands (77\%) showed weakness, and 292 hands (96\%) had nocturnal paresthesias.

Six months after surgery 260 hands $(85 \%)$ were reported by patients to show improvement in function and 272 hands $(89 \%)$ were relieved of their preoperative nocturnal paresthesias allowing patients to sleep through the night.

The patients without diabetes gave the following responses on the questionnaire: $203(67 \%)$ of 305 hands were rated as excellent; 72 (24\%) were rated as good; $15(5 \%)$ were rated as fair; and $15(5 \%)$ were rated as poor (Fig. 1). Overall patients reported that 275 (90\%) of 305 hands had a satisfactory outcome. 
Patients with certain systemic processes that predispose peripheral nerves to the effects of compression are more likely than those in the general population to develop one of two common entrapment neuropathies involving the median nerve in the carpal tunnel and the ulnar nerve at the elbow. This predisposition has been found in patients with diabetes mellitus, hypothyroidism, renal failure with chronic hemodialysis, rheumatoid arthritis, cervical arthritis, tenosynovitis, amyloidosis, acromegaly, and in pregnant women.[7,11]

Some entrapment syndromes can be attributed to the fact that these processes are associated with the addition of soft tissue to the contents of the carpal tunnel or cubital tunnel.[1,6,16] This is obvious when considering acromegaly, thyroid dysfunction, pregnancy, amyloidosis, rheumatoid arthritis, and tenosynovitis, in which a deposition of soft tissue or even fluid can compromise the contents of the carpal tunnel. It is less obvious, however, in diabetes, chronic renal dialysis, and cervical arthritis, when the effect is not clearly related to an increase in the soft-tissue contents of the carpal tunnel.

Patients with longstanding diabetes mellitus and polyneuropathy show characteristic pathological changes in their peripheral nerves. There is loss of myelinated and unmyelinated fibers, segmental demyelination, proliferation of Schwann cells, and an increase in intrinsic fibrous tissue within the nerve.[10] In patients who have been recently diagnosed as diabetic, however, the neuropathy can manifest itself as reduction in nerve conduction velocities without overt anatomical abnormalities in the nerve itself.[2] In these patients, something other than structural abnormalities must account for the changes in conduction velocities.[10]

In some patients with diabetes, particularly those with polyneuropathy, the nerve is injured throughout its course. This injury is not clearly a primary axonal or Schwann cell insult, nor is it necessarily a vascular event.[2] Whatever the mechanism, there is increased susceptibility to further compression of the nerve at common sites of entrapment.[5,9] If one considers the initial diabetic insult to the nerve as a form of crush syndrome, as suggested by Dellon and coworkers, $[5,16]$ then we should expect the nerve to respond to surgical release just as we do in those nondiabetic patients with tandem lesions,[16] such as cervical radiculopathy and CTS.[5] We believe our results confirm this hypothesis.

The results of our studies show that median nerve decompression by division of the transverse carpal ligament in patients with diabetes is nearly as successful as it is in patients without diabetes. This is true both for insulin-dependent and noninsulin-dependent patients. Even in those insulin-dependent patients with diffuse polyneuropathy or multiple nerve entrapment neuropathies, the results of surgical decompression are still very good. These patients should be considered surgical candidates despite the coexistence of diabetes. Our results, and those of others, $[3,8]$ suggest that patients with diabetes should be considered for CTR surgery just as those without diabetes. Early treatment produces the best outcome.

\section{Acknowledgment}

We are grateful to Lynn Morawski for maintaining our database and retrieval system and for her diligence and hard work on this paper to its final goal of publication.

\section{References}

1. Bleecker ML, Bohlman M, Moreland R, et al: Carpal tunnel syndrome: role of carpal tunnel size. Neurology 35:1599-1604, 1985 
2. Brown MJ, Asbury AK: Diabetic neuropathy. Ann Neurol 15:2-12, 1984

3. Dellon AL: Treatment of symptomatic diabetic neuropathy by surgical decompression of multiple peripheral nerves. Plast Reconstr Surg 4:689-699, 1992

4. Dellon AL, Mackinnon SE: Basic scientific and clinical applications of peripheral nerve regeneration. Surg Annu 20:59-100, 1988

5. Dellon AL, Mackinnon SE, Seiler WA IV: Susceptibility of the diabetic nerve to chronic compression. Ann Plast Surg 20:117-119, 1988

6. Dorwart BB: Carpal tunnel syndrome: a review. Semin Arthrit Rheum 14:134-140, 1984

7. Faithfull DK, Mori DH, Ireland J: The micropathology of the typical carpal tunnel syndrome. J Hand Surg (Br) 11:131-132, 1986

8. Greene DA, De Jesus PV Jr, Winegrad AI: Effects of insulin and dietary myoinositol on impaired peripheral motor nerve conduction velocity in acute streptozotocin diabetes. J Clin Invest 55:1326-1336, 1975

9. Massey EW, Pleet AB: Compression injury of the sciatic nerve during a prolonged surgical procedure in a diabetic patient. J Am Geriatr Soc 28:188-189, 1980

10. O'Brian JT, Massey EW: Mononeuropathy in diabetes mellitus: a phenomenon easily overlooked. Postgrad Med 65:128, 130-132, 135-136 passim, 1979

11. Ozaki I, Baba M, Matsunaga M, et al: Deleterious effect of the carpal tunnel on nerve conduction polyneuropathy. Electromyogr Clin Neurophysiol 28:301-306, 1988

12. Pagnanelli DM, Barrer SJ: Carpal tunnel syndrome: surgical treatment using the Paine retinaculotome. J Neurosurg 75:77-81, 1991

13. Paine KWE, Polyzoidis KS: Carpal tunnel syndrome. Decompression using the Paine retinaculotome. J Neurosurg 59:1031-1036, 1983

14. Powell HC: Diabetes: effects on the nervous system, in Adelman G (ed): Encyclopedia of Neuroscience. Boston: Birkhauser, 1987, pp 323-333

15. Rydevik B, Lundborg G, Bagge U: Effects of graded compression on interaneural blood flow. An in vivo study on rabbit tibial nerve. J Hand Surg (Am) 6:3-12, 1981

16. Seiler R, Schlegel R, Mackinnon SE, et al: Double crush syndrome: experimental model in the rat. Surg Forum 34:597, 1983

17. Tomlinson DR, Moriarty RJ, Mayer JH: Prevention and reversal of defective axonal transport and motor nerve conduction velocity in rats with experimental diabetes by treatment with the aldose reductase inhibitor Sorbinil. Diabetes 33:470-476, 1984

Manuscript received May 19, 1997.

Accepted in final form June 10, 1997. 
Address reprint requests to: David M. Pagnanelli, M.D., 1584 Old York Road, Abington, Pennsylvania 19001. 